\title{
Molecular mechanisms of [18F]fluorodeoxyglucose accumulation in liver cancer
}

\author{
KUNIHIKO IZUISHI $^{1,4}$, YUKA YAMAMOTO ${ }^{2}$, HIROHITO MORI ${ }^{3}$, RIKO KAMEYAMA $^{2}$, \\ SHINTARO FUJIHARA ${ }^{3}$, TSUTOMU MASAKI $^{3}$ and YASUYUKI SUZUKI ${ }^{1}$ \\ Departments of ${ }^{1}$ Gastroenterological Surgery, ${ }^{2}$ Radiology and ${ }^{3}$ Internal Medicine of Gastroenterology \\ and Neurology, Faculty of Medicine, Kagawa University, Miki, Kita, Kagawa 761-0793; \\ ${ }^{4}$ Department of Gastroenterological Surgery, Federation of Public Services and Affiliated Personnel \\ Aid Associations, Takamatsu Hospital, Takamatsu, Kagawa 760-0018, Japan
}

Received September 10, 2013; Accepted October 25, 2013

DOI: $10.3892 /$ or.2013.2886

\begin{abstract}
To elucidate the molecular mechanisms underlying the insufficient sensitivity in the detection of hepatocellular carcinoma (HCC) by [18F] 2-fluoro-2-deoxy-D-glucose positron emission tomography (FDG-PET), the characteristics of glucose metabolism-related protein expression in HCC were examined in liver metastasis from colorectal cancer (Meta). Thirty-four patients (14 Meta and $20 \mathrm{HCC}$ ) who underwent FDG-PET and hepatectomy were studied. The relationships between the maximum standardized uptake value (SUV) in tumors and the mRNA expression of glucose metabolismrelated proteins [hexokinase (HK), glucose transporter 1 (GLUT1), and glucose-6-phosphatase (G6Pase)] and proliferating cell nuclear antigen (PCNA) were examined in snap-frozen specimens with quantitative PCR. Tumor detection rates were lower in HCC $(15 / 20)$ compared to Meta (13/14) patients. HK and GLUT1 expression was lower and G6Pase expression was higher in HCC compared to Meta. In particular, GLUT1 overexpression was 92-fold in Meta and 11-fold in HCC compared to the surrounding liver. The SUV correlated with GLUT1 and PCNA expression in HCC, but not Meta patients. Of note, four cases of poorly differentiated (P/D) HCC compared to moderately differentiated (M/D) HCC produced completely different results for FDG uptake (SUV, 14.4 vs. 4.0) and mRNA expression (G6Pase expression, 0.007 vs. 1.5). Variations in the expression of glucose metabolism-related enzymes between HCC and Meta patients are attributed to origin or degree of differentiation. Low FDG
\end{abstract}

Correspondence to: Dr Kunihiko Izuishi, Department of Gastroenterological Surgery, Federation of Public Services and Affiliated Personnel Aid Associations, Takamatsu Hospital, 4-18 Tenjinmae, Takamatsu, Kagawa 760-0018, Japan

E-mail: izuishi@kkr-ta-hp.gr.jp

Key words: 18-fluorodeoxyglucose, positron emission tomography, hepatocellular carcinoma, liver metastasis, glucose transporter-1 uptake in M/D HCC reflected low GLUT1 and high G6Pase expression, while high FDG accumulation in P/D HCC could reflect increased GLUT1 and decreased G6Pase expression. These results may explain why M/D HCC is not detected as sensitively by FDG-PET.

\section{Introduction}

Recent advancements in imaging techniques include positron emission tomography (PET) with [18F] 2-fluoro-2-deoxyD-glucose (FDG), which has been widely used for clinical molecular imaging $(1,2)$. Several studies have described its usefulness in detecting primary tumors and metastases for several types of cancer $(3,4)$. In particular, previous studies reported the increased FDG uptake in hepatocellular carcinoma (HCC) lesions (5). However, the sensitivity of HCC detection with FDG-PET is only $50-55 \%$ (6-8), which is significantly lower than the $98 \%$ detection rate for liver metastasis from colorectal cancer (9). FDG-PET is therefore less useful for detecting primary HCC lesions than conventional ultrasonography or computed tomography.

Nevertheless, FDG-PET has received considerable attention as a new modality with the ability to estimate the malignant potential of tumors. Several studies have demonstrated that FDG accumulation reflects tumor aggressiveness and predicts poor survival (10-12). Therefore, FDG-PET has been used to evaluate the malignant potential of tumors after chemotherapy (13). New molecular-targeted cancer therapies [such as vascular endothelial growth factor inhibitor (bevacizumab) and epidermal growth factor receptor inhibitor (cetuximab)] require new methods for monitoring treatment progress (14), as they exert cytostatic instead of the cytoreductive effects of traditional chemotherapy. Compared with computed tomography, FDG-PET provides information on tumor response to bevacizumab therapy earlier (15). The multiple kinase inhibitor sorafenib is a new molecular-targeted therapy approved for the treatment of HCC. Given the strong FDG uptake observed in HCC tumors, FDG-PET may be useful for evaluating the cytostatic effects of this type of therapy (16).

The molecular mechanisms involved in FDG imaging relate to its uptake by glucose transporter 1 (GLUT1) and 
metabolism by hexokinase (HK) and glucose-6-phosphatase (G6Pase). FDG accumulates in malignant cells via GLUT1 transport and HK phosphorylation, as shown in Fig. 1 (17). G6Pase, a gluconeogenesis enzyme strongly expressed in the liver $(17,18)$, counteracts HK phosphorylation by converting glucose-6-phosphate to glucose. High G6Pase levels therefore reduce FDG accumulation by accelerating the conversion of FDG-6-phosphate to FDG, leading to its release from cells.

Although previous studies have evaluated the molecular mechanisms underlying FDG-PET, most relied on nonquantitative immunohistochemistry analysis such as positive or negative staining. Since interpretation of these results is subjective, it is difficult to determine the precise relationship between the standardized uptake value (SUV) and glucose metabolism-related protein levels. To determine the mechanisms responsible for low FDG-PET efficacy in HCC, we evaluated glucose metabolism-related protein expression in $\mathrm{HCC}$ with that of another type of liver cancer (liver metastasis from colorectal cancer) using quantitative reverse-transcription polymerase chain reaction (qRT-PCR). We then evaluated the relationship between protein expression and SUV in each type of liver cancer.

\section{Materials and methods}

Patients. The present study included 34 patients with liver cancer (21 male and 13 female; mean age \pm SEM, 67.6 \pm 1.7 years) who underwent FDG-PET prior to surgery in our hospital. Of these patients, 20 had HCC (HCC group) and 14 had liver metastasis from colorectal cancer (Meta group). Specimens consisting of the tumors and surrounding normal liver tissue were immediately snap-frozen in liquid nitrogen after surgical resection and stored at $-80^{\circ} \mathrm{C}$. These specimens were later thawed for isolation and analysis of mRNA levels. Of the 20 HCC cases, 16 were moderately differentiated (M/D) HCC, and four were poorly differentiated (P/D) HCC. The protocol was approved by the institutional review board and all patients provided written informed consent.

PET imaging. FDG-PET images were acquired with a PET scanner (ECAT EXACT $\mathrm{HR}^{+}$, Siemens/CTI, Knoxville, TN, USA). Patients fasted at least $5 \mathrm{~h}$ before FDG injection. Images were reviewed on a Sun Microsystems workstation (Siemens/CTI) along transverse, coronal, and sagittal planes with maximum intensity projection images. The images were interpreted independently by two experienced nuclear medicine physicians blinded to the clinical data. Tumor lesions were identified as areas of focally increased FDG uptake exceeding that of the surrounding normal tissue. A region of interest was placed over each lesion to include the highest levels of radioactivity. The maximum SUV was calculated with the following formula: SUV $=\mathrm{cdc} /(\mathrm{di} / \mathrm{w})$, where $\mathrm{cdc}$ is the decay-corrected tracer tissue concentration $(\mathrm{Bq} / \mathrm{g})$, di is the injected dose $(\mathrm{Bq})$, and $\mathrm{w}$ is the body weight of the patient $(\mathrm{g})$.

Immunohistochemical staining. Immunohistochemical staining was performed to determine levels of GLUT1 and GLUT2 in the liver cancer specimens. Briefly, resected specimens were fixed in $10 \%$ buffered formalin solution, embedded in paraffin, and sectioned $(4 \mu \mathrm{m})$. Slides were incubated

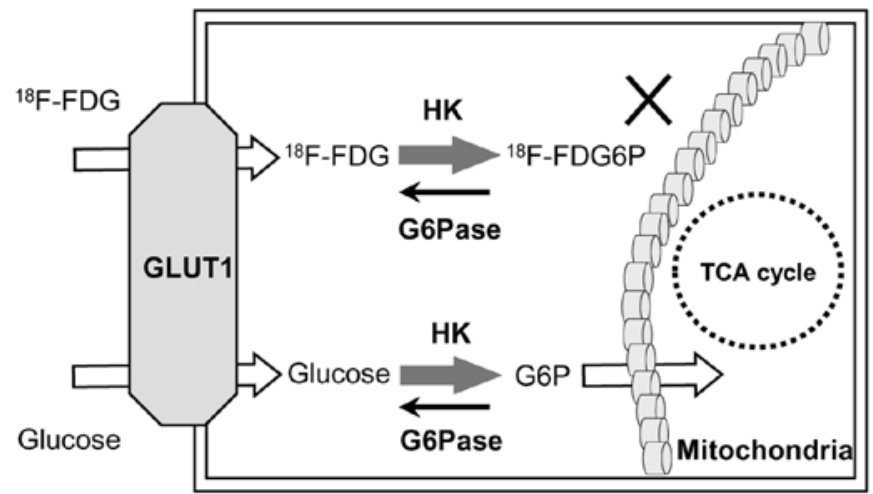

Figure 1. Schematic for the metabolic trapping of [18F]fluorodeoxyglucose in tumor cells. ${ }^{18} \mathrm{~F}-\mathrm{FDG},{ }^{18} \mathrm{~F}$-2-fluoro-2-deoxy-D-glucose; ${ }^{18} \mathrm{~F}$-FDG6P, ${ }^{18} \mathrm{~F}-2$ fluoro-2-deoxy-D-glucose-6-phosphate; GLUT1, glucose transporter 1; G6P, glucose-6-phosphate; G6Pase, glucose-6-phosphatase; HK, hexokinase; TCA, tricarboxylic acid.
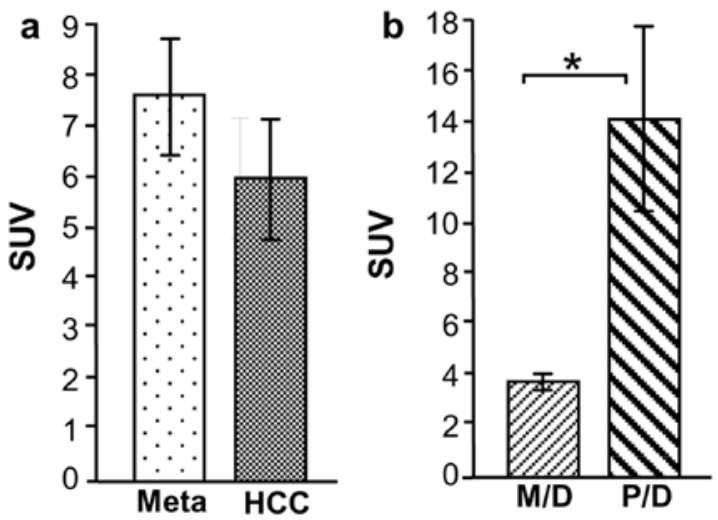

Figure 2. Standardized uptake value (SUV) of [18F]fluorodeoxyglucose in liver cancer. (a) The mean SUV in hepatocellular carcinoma (HCC) was similar to that of metastatic tumors from colorectal cancer (Meta). (b) The mean SUV of poorly differentiated (P/D) HCC $(n=4)$ was higher than that of moderately differentiated (M/D) HCC $(n=16)$.

overnight at room temperature with primary rabbit polyclonal antibodies against GLUT1 or GLUT2 (1:200 dilution). Avidinbiotin-peroxidase complex staining was performed according to the manufacturer's instructions (Santa Cruz Biotechnology, Santa Cruz, CA, USA). Finally, the nuclei were counterstained with hematoxylin.

$q R T-P C R$. Total RNA was isolated from the specimens by guanidinium isothiocyanate-acid phenol extraction and quantified by absorbance at $260 \mathrm{~nm}$. Reverse transcription was carried out with $1 \mu \mathrm{g}$ RNA, and the resulting cDNA was analyzed by real-time PCR with Power SYBR Green PCR Master Mix and the ABI Prism 7000 (Applied Biosystems, Foster, CA, USA). Target-specific oligonucleotide primers and probes were previously described $(19,20)$. As endogenous control, the amplification of $18 \mathrm{~S}$ rRNA was used. The relative value of mRNA expression indicates the ratio of the mRNA expression to mean mRNA levels in normal liver after $18 \mathrm{~S}$ rRNA normalization. Primers and probes for 18S rRNA were obtained in a Pre-Developed TaqMan Assay Reagent kit (Applied Biosystems, Stockholm, Sweden). 


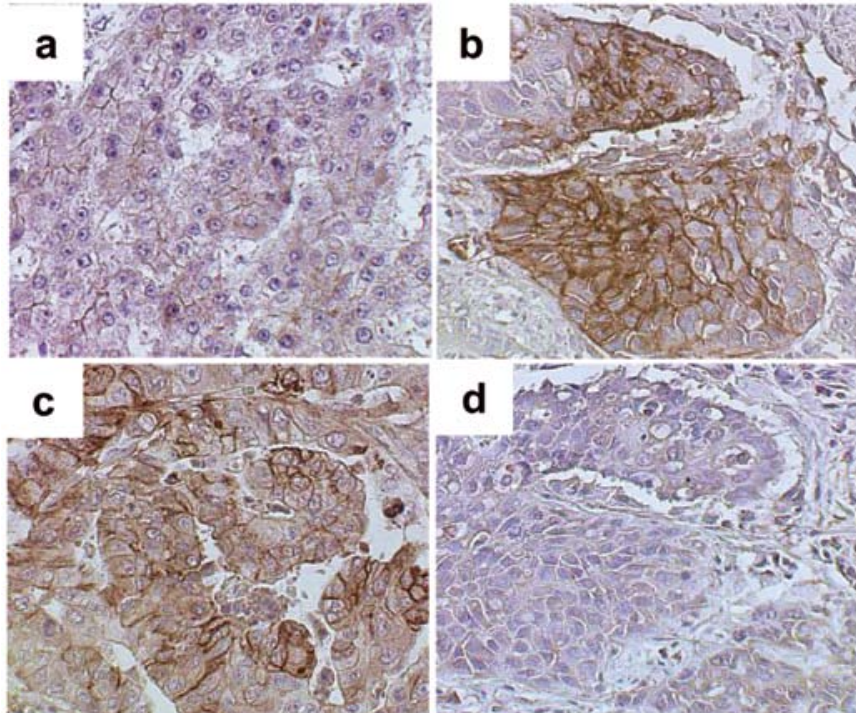

Figure 3. Immunohistochemical analysis of membrane-bound glucose transporter in liver cancer. Weak immunostaining of glucose transporter (GLUT)1 was observed in the cell membrane of (a) moderately differentiated hepatocellular carcinoma cells. Strong GLUT1 staining was observed in (b) poorly differentiated hepatocellular carcinoma and (c) metastatic tumors from colorectal cancer. Only weak GLUT2 staining was observed in (d) poorly differentiated hepatocellular carcinoma.

Statistical analysis. Results are expressed as mean \pm SEM. SUV values were compared by Student's t-test. Multiple one-way analysis of variance (ANOVA) was used to assess differences in mRNA levels. Correlation analyses were performed with the Spearman's rank correlation test. P-values $<0.05$ were considered to indicate statistically significant differences.

\section{Results}

Tumor detection and SUV with FDG-PET.FDG-PET detected 15/20 HCC lesions (SUV, 6.1 \pm 1.2 ) and 13/14 Meta lesions
(SUV, 7.7 \pm 1.1 ) (Fig. 2a). Of the HCC cases, all four P/D HCC lesions were detected by FDG-PET with a high mean SUV (14.4 \pm 3.7$)$. By contrast, 11/16 M/D HCC lesions were detected by FDG-PET, and the mean SUV (4.0 \pm 0.3$)$ was significantly lower than that of P/D HCC (P<0.0001) (Fig. 2b).

Immunohistochemical analysis of GLUT1 and GLUT2. To elucidate the molecular mechanisms underlying FDG accumulation in liver cancer, tumor levels of GLUT1 and GLUT2 were evaluated. The M/D HCC cell membranes showed only weak staining with GLUT1 antibody (Fig. 3a), but P/D HCC and Meta specimens showed relatively strong GLUT1 membrane staining (Fig. 3b and c). Staining with the GLUT2 antibody was not observed in P/D HCC cells (Fig. 3d).

HK1, HK2, GLUT1, GLUT2, and G6Pase mRNA levels in liver cancer and normal liver tissue. Based on the immunostaining results, the tissue specimens were evaluated by qRT-PCR to determine expression of glucose metabolism-related proteins GLUT1, GLUT2, HK1, HK2, and G6Pase. Compared with normal liver tissue, HK1 and HK2 mRNA levels were higher in Meta specimens $(\mathrm{P}<0.01)$, but were unchanged in HCC specimens (Fig. 4a and b). Similarly, GLUT1 expression was higher in Meta specimens than in normal liver tissue $(\mathrm{P}<0.01)$, but was not significantly higher in HCC specimens (Fig. 4c). By contrast, GLUT2 and G6Pase mRNA levels were considerably lower in Meta specimens $(\mathrm{P}<0.01$ and $\mathrm{P}<0.05$, respectively) than in normal tissue, but were unchanged in HCC specimens (Fig. 4d and e).

HK1, HK2, GLUT1, GLUT2, and G6Pase mRNA levels according to HCC differentiation. Expression of glucose metabolism-related proteins in M/D HCC and P/D HCC was also compared, revealing a considerable difference in expression patterns. Although HK1 mRNA levels were similar in the two groups, HK2 and GLUT1 mRNA levels were higher in P/D HCC compared with M/D HCC and normal liver tissue
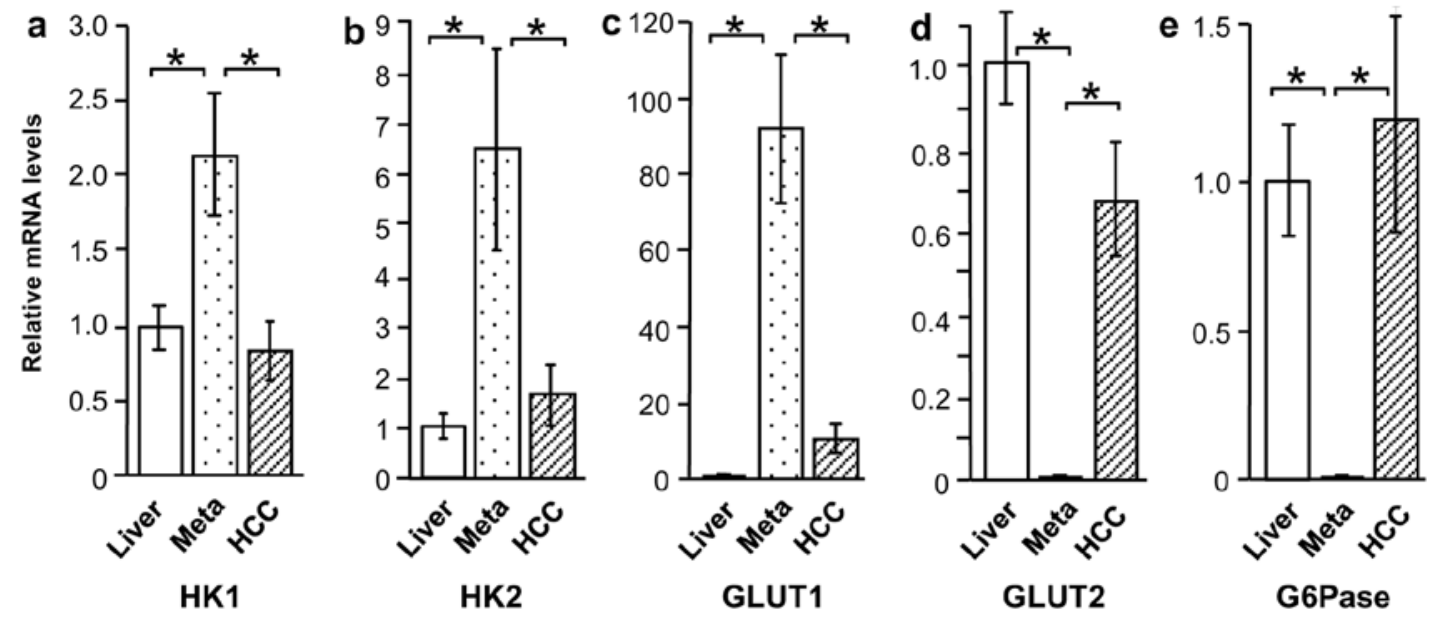

Figure 4. Expression of glucose metabolism-related proteins in liver cancer and normal liver tissue. (a) Compared with normal liver tissue, hexokinase 1 (HK1) mRNA levels were higher in metastatic tumors (Meta) but were unchanged in hepatocellular carcinoma (HCC). (b) Compared with normal liver tissue, HK2 mRNA levels were higher in Meta specimens but were unchanged in HCC. (c) Compared with normal liver tissue, glucose transporter 1 (GLUT1) mRNA levels were 92-fold higher in Meta specimens. GLUT1 mRNA levels appeared to be 11-fold higher in HCC, but this increase was not significant. (d) Compared with normal liver tissue, GLUT2 expression was significantly lower in Meta specimens but was not detectable in HCC. (e) Compared with normal liver tissue, glucose-6-phosphatase (G6Pase) expression was decreased in Meta but was unchanged in HCC. "P<0.05 (ANOVA). 


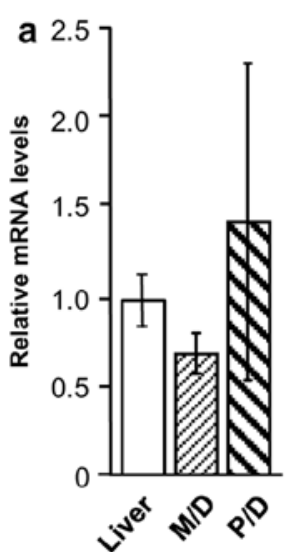

HK1

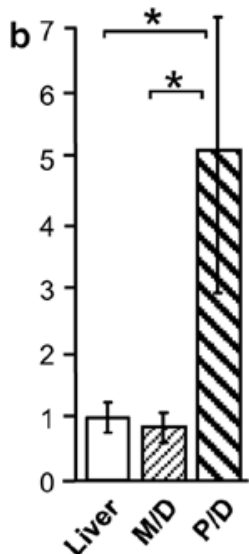

HK2

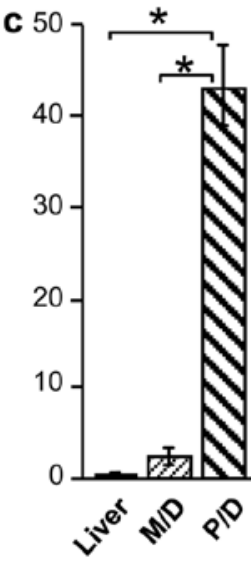

GLUT1

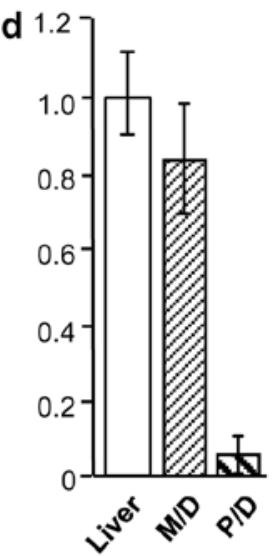

GLUT2

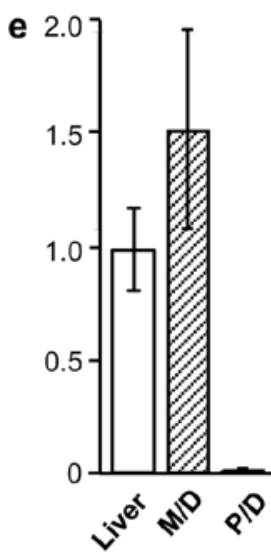

G6Pase

Figure 5. Expression of glucose metabolism-related proteins in moderately differentiated and poorly differentiated hepatocellular carcinoma. (a) Hexokinase 1 (HK1) mRNA levels in hepatocellular carcinomas (HCCs) were similar to those of normal liver tissue. (b) HK2 expression was significantly higher in poorly differentiated (P/D) HCC compared with normal liver and moderately differentiated (M/D) HCC. (c) Glucose transporter 1 (GLUT1) expression was significantly higher in P/D HCC compared with normal liver and M/D HCC. (d) GLUT2 appeared to be lower in P/D HCC, but this difference was not significant. (e) Glucose-6-phosphatase (G6Pase) appeared to be lower in P/D HCC, but this difference was not significant. "P<0.05 (ANOVA).
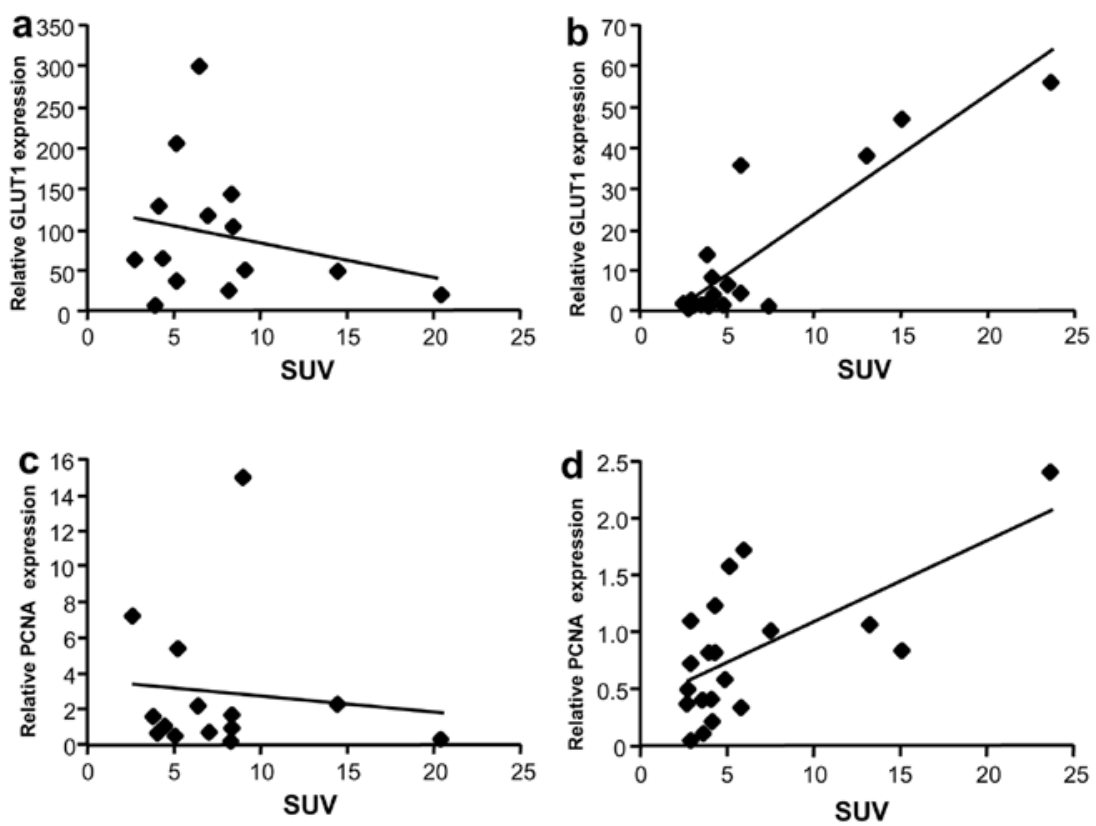

Figure 6. Relationship between mean standardized uptake value (SUV) and expression of glucose transporter 1 (GLUT1) or proliferating cell nuclear antigen in liver cancer. (a) SUV and GLUT1 expression were not associated in liver metastasis from colorectal cancer, (b) but a correlation was found in hepatocellular carcinoma. (c) SUV and proliferating cell nuclear antigen (PCNA) expression were not associated in liver metastasis from colorectal cancer, (d) but a significant correlation was found in hepatocellular carcinoma.

$(\mathrm{P}<0.01)$ (Fig. 5a-c). By contrast, GLUT2 and G6Pase appeared to be lower in P/D HCC (18- and 142-fold, respectively), but this increase was not significant (Fig. 5d and e).

Correlation between SUVandmRNA levels of GLUT1 or proliferating cell nuclear antigen. Since GLUT1 is overexpressed in liver cancer, the relationship between GLUT1 mRNA expression and SUV was evaluated. Although GLUT1 mRNA levels were not associated with SUV in the Meta group (Fig. 6a), a correlation was observed in the HCC group ( $\mathrm{rs}=0.69, \mathrm{P}=0.002$ ) (Fig. 6b). To further evaluate the relationship between SUV and tumor growth, mRNA levels of a proliferation marker, proliferating cell nuclear antigen (PCNA), were analyzed. No correlation was observed between PCNA mRNA levels and SUV in Meta specimens (Fig. 6c), but a weak correlation was observed in HCC specimens (Fig. 6d, rs=0.58, $\mathrm{P}=0.01$ ).

\section{Discussion}

FDG-PET is used for diagnosis and tumor staging essential for appropriate cancer management. However, FDG accumulation is low in HCC tumors compared with other types of cancer, such as lung cancer or malignant lymphoma $(21,22)$. Therefore, we investigated the mechanisms underlying FDG 
accumulation by comparing two major forms of liver cancer HCC and colorectal cancer liver metastasis (Meta). We found that SUV correlated with GLUT1 expression in HCC but not Meta specimens. This finding may be due to the lower GLUT1 expression in HCC tumors, particularly M/D HCC cases. Compared with surrounding normal liver tissue, GLUT1 levels were 11-fold higher in HCC specimens and 92-fold higher in Meta specimens. Thus, the number of GLUT1 receptors in Meta tumors likely exceeds the requirements for maximum FDG transport, accounting for the lack of association between SUV and GLUT1 expression. The lower GLUT1 levels in HCC suggest that this transporter may still be a rate-limiting factor for FDG uptake, with the SUV dependent on GLUT1 increase.

SUV also correlated with tumor growth (assessed by PCNA expression) in HCC specimens but not Meta specimens. The increased energy provided by GLUT1 overexpression likely plays a role in both HCC and Meta tumor growth; therefore, the reason for the contrasting PCNA results is unclear. However, the PCNA expression in Meta specimens was consistent with a previous study (20). These results indicate that SUV cannot always estimate tumor growth or prognosis in the patients with liver metastasis of colorectal cancer.

GLUT1 is responsible for the cell basal glucose requirements. The role of GLUT1 as the primary transporter in FDG uptake for HCC is controversial as there are $>10$ GLUT family isoforms, which are expressed in a tissue-specific manner and have specific roles (23). Paudyal et al (24) reported GLUT2 expression in $71 \%$ of HCCs and GLUT1 expression in only $16 \%$ of HCCs. Roh et al (25) reported that GLUT1 was expressed in $81.3 \%$ of cholangiocarcinomas but only $4.5 \%$ of HCCs. Taken together, these immunohistochemical studies indicate that GLUT1 overexpression does not occur in all HCCs (24). Our immunohistochemical results showed relatively strong GLUT2 expression in HCC specimens; however, the qRT-PCR results demonstrated that GLUT2 expression was similar to that of the surrounding normal liver tissue. By contrast, GLUT1 was clearly overexpressed in HCC in the present study, with levels $\sim 11$-fold higher than those of the surrounding normal tissue.

GLUT2 is expressed in hepatocytes, pancreatic $\beta$ cells, and the basolateral membranes of intestinal and renal epithelial cells (26). In the liver, GLUT2 expressed on hepatocyte membranes allows for the bidirectional glucose transport, allowing glucose flux in and out of cells (27). Unlike other GLUT family members, GLUT2 is important for sensing glucose concentration in the pancreas, intestinal glucose uptake, glucose resorption by the kidney, and glucose uptake and release in the liver (28). In humans, the physiological glucose concentration is $5.6 \mathrm{mmol} / \mathrm{l}$, and the GLUT1 and GLUT2 Michaelis constants are $<20 \mathrm{mmol} / 1$ and $40 \mathrm{mmol} / 1$, respectively (28). Thus, GLUT1 has a higher affinity for glucose than GLUT2, indicating that GLUT1 overexpression may provide a major advantage regarding glucose uptake in cancer cells in the liver.

Previous studies reported that HK overexpression also contributes to strong FDG uptake in HCC $(29,30)$. In this study, the mRNA levels of HK1 and HK2 were higher in Meta specimens than in HCC specimens. The difference in $\mathrm{HKs}$ was relatively small compared with the large difference in GLUT1 expression of the Meta or HCC. However, this transport protein may still play a role in FDG uptake.
A molecular mechanism was also required to explain differences in FDG uptake between M/D HCC and P/D HCC specimens. However, it is important to note that the P/D HCC results were derived from a small number of cases and showed considerable variability in the results. Torizuka et al (17) reported that G6Pase activity was lower in $\mathrm{P} / \mathrm{D} \mathrm{HCC}$ than in well-differentiated HCC or M/D HCC. Our mRNA expression data were consistent with these results. In addition, GLUT and HK mRNA levels were similar in P/D HCC and Meta specimens. Of note, the G6Pase mRNA level of M/D HCC specimens was 218-fold greater than that of P/D HCC. Overexpression of G6Pase may allow the FDG trapped in cells to be released into the bloodstream. The SUV of HCC tumors may thus reflect how well HCC retains the nature of normal liver tissue. High FDG accumulation in P/D HCC may reflect increased GLUT1 expression and decreased G6Pase expression compared with M/D HCC, which would explain why differentiated HCC shows lower FDG accumulation compared with other malignancies.

In conclusion, the present study suggests that the molecular mechanism for FDG-PET molecular imaging depends on GLUT1 and G6Pase expression. Low GLUT1 and high G6Pase expression contribute to low FDG uptake in HCC tumors, preventing efficient tumor detection. A pattern of high GLUT1 and low G6Pase expression in P/D HCC facilitated FDG uptake similar to that of liver metastasis from colorectal cancer. Finally, GLUT2 expression, while important in normal hepatocytes, did not contribute to FDG uptake in either type of liver cancer.

\section{Acknowledgements}

The authors are grateful to all the clinical staff who cared for these patients. We also thank Dr Shoji Kimura for his reliable experimental suggestions.

\section{References}

1. Fass L: Imaging and cancer. Mol Oncol 2: 115-152, 2008.

2. Mawlawi $\mathrm{O}$ and Townsend DW: Multimodality imaging: an update on PET/CT technology. Eur J Nucl Med Mol Imaging 36 (Suppl 1): 15-29, 2009.

3. Schaefer O and Langer M: Detection of recurrent rectal cancer with CT, MRI and PET/CT. Eur Radiol 17: 2044-2054, 2007.

4. Sugiyama M, Sakahara H, Torizuka T, Kanno T, Nakamura F, Futatsubashi M and Nakamura S: 18F-FDG PET in the detection of extrahepatic metastases from hepatocellular carcinoma. J Gastroenterol 39: 961-968, 2004.

5. Iwata Y, Shiomi S, Sasaki N, et al: Clinical usefulness of positron emission tomography with fluorine-18-fluorodeoxyglucose in the diagnosis of liver tumors. Ann Nucl Med 14: 121-126, 2000.

6. Khan MA, Combs CS, Brunt EM, et al: Positron emission tomography scanning in the evaluation of hepatocellular carcinoma. J Hepatol 32: 792-797, 2000.

7. Trojan J, Schroeder O, Raedle J, et al: Fluorine-18 FDG positron emission tomography for imaging of hepatocellular carcinoma. Am J Gastroenterol 94: 3314-3319, 1999.

8. Nagaoka S, Itano S, Ishibashi M et al: Value of fusing PET plus $\mathrm{CT}$ images in hepatocellular carcinoma and combined hepatocellular and cholangiocarcinoma patients with extrahepatic metastases: preliminary findings. Liver Int 26: 781-788, 2006.

9. Akiyoshi T, Oya M, Fujimoto Y, et al: Comparison of preoperative whole-body positron emission tomography with MDCT in patients with primary colorectal cancer. Colorectal Dis 11: 464-469, 2009

10. Seo S, Hatano E, Higashi T, et al: Fluorine-18 fluorodeoxyglucose positron emission tomography predicts tumor differentiation, P-glycoprotein expression, and outcome after resection in hepatocellular carcinoma. Clin Cancer Res 13: 427-433, 2007. 
11. Lee JD, Yun M, Lee JM, et al: Analysis of gene expression profiles of hepatocellular carcinomas with regard to $18 \mathrm{~F}$-fluorodeoxyglucose uptake pattern on positron emission tomography. Eur J Nucl Med Mol Imaging 31: 1621-1630, 2004.

12. Hatano E, Ikai I, Higashi T, et al: Preoperative positron emission tomography with fluorine-18-fluorodeoxyglucose is predictive of prognosis in patients with hepatocellular carcinoma after resection. World J Surg 30: 1736-1741, 2006.

13. Byström P, Berglund A, Garske U, et al: Early prediction of response to first-line chemotherapy by sequential [18F]-2-fluoro2-deoxy-D-glucose positron emission tomography in patients with advanced colorectal cancer. Ann Oncol 20: 1057-1061, 2009.

14. Chau I and Cunningham D: Treatment in advanced colorectal cancer: what, when and how? Br J Cancer 100: 1704-1719, 2009.

15. de Langen AJ, van den Boogaart V, Lubberink M, et al: Monitoring response to antiangiogenic therapy in non-small cell lung cancer using imaging markers derived from PET and dynamic contrast-enhanced MRI. J Nucl Med 52: 48-55, 2011.

16. Lee JH, Park JY, Kim do Y, et al: Prognostic value of 18F-FDG PET for hepatocellular carcinoma patients treated with sorafenib. Liver Int 31: 1144-1149, 2011.

17. Torizuka T, Tamaki N, Inokuma T, et al: In vivo assessment of glucose metabolism in hepatocellular carcinoma with FDG-PET. J Nucl Med 36: 1811-1817, 1995.

18. Caracó C, Aloj L, Chen LY, Chou JY and Eckelman WC: Cellular release of [18F]2-fluoro-2-deoxyglucose as a function of the glucose-6-phosphatase enzyme system. J Biol Chem 275: 18489-18494, 2000

19. Kameyama R, Yamamoto Y, Izuishi K, Sano T and Nishiyama $Y$ Correlation of 18F-FLT uptake with equilibrative nucleoside transporter-1 and thymidine kinase-1 expressions in gastrointestinal cancer. Nucl Med Commun 32: 460-465, 2011.

20. Izuishi K, Yamamoto Y, Sano T, et al: Molecular mechanism underlying the detection of colorectal cancer by 18F-2-fluoro-2deoxy-d-glucose-positron emission tomography. J Gastrointest Surg 16: 394-400, 2012.
21. Murakami K: FDG-PET for hepatobiliary and pancreatic cancer: Advances and current limitations. World J Clin Oncol 2: 229-236, 2011.

22. Lin WY, Tsai SC and Hung GU: Value of delayed 18F-FDG-PET imaging in the detection of hepatocellular carcinoma. Nucl Med Commun 26: 315-321, 2005.

23. Zhao FQ and Keating AF: Functional properties and genomics of glucose transporters. Curr Genomics 8: 113-128, 2007.

24. Paudyal B, Paudyal P, Oriuchi N, Tsushima Y, Nakajima T and Endo K: Clinical implication of glucose transport and metabolism evaluated by 18 F-FDG PET in hepatocellular carcinoma. Int J Oncol 33: 1047-1054, 2008.

25. Roh MS, Jeong JS, Kim YH, Kim MC and Hong SH: Diagnostic utility of GLUT1 in the differential diagnosis of liver carcinomas. Hepatogastroenterology 51: 1315-1318, 2004.

26. Wood IS and Trayhurn P: Glucose transporters (GLUT and SGLT): expanded families of sugar transport proteins. Br J Nutr 89: 3-9, 2003

27. Brown GK: Glucose transporters: structure, function and consequences of deficiency. J Inherit Metab Dis 23: 237-246, 2000.

28. Leturque A, Brot-Laroche E, Le Gall M, Stolarczyk E and Tobin V: The role of GLUT2 in dietary sugar handling. J Physiol Biochem 61: 529-537, 2005

29. Ong LC, Jin Y, Song IC, Yu S, Zhang K and Chow PK: 2-[18F]-2deoxy-D-glucose (FDG) uptake in human tumor cells is related to the expression of GLUT-1 and hexokinase II. Acta Radiol 49: $1145-1153,2008$

30. Paudyal B, Oriuchi N, Paudyal P, et al: Clinicopathological presentation of varying 18F-FDG uptake and expression of glucose transporter 1 and hexokinase II in cases of hepatocellular carcinoma and cholangiocellular carcinoma. Ann Nucl Med 22: 83-86, 2008. 